\title{
Periodontitis and Diabetes- A Two Way Connection
}

\author{
Dr. Satya akhila ${ }^{1}$, Dr. sankari malaiappan ${ }^{2}$ \\ ${ }^{I}$ ( resident, department of periodontics / saveetha university, india) \\ ${ }^{2}$ (senior proffessor, department of periodontics / saveetha university, india)
}

\begin{abstract}
Periodontal disease is an entity of localised infection that involves tooth supporting tissues, the structures that make up the periodontium. The disease being microbial in origin, the bacterial flora from the bio film invades the periodontal connective tissue and alveolar bone causing loosening of tooth. Diabetes being a metabolic disorder also has an inflammatory origin which brings a strong correlation between the two complex diseases. Both the diseases are chronic affecting large population worldwide. It's been proposed that periodontitis may cause changes in systemic physiology. And a systemic disease like diabetes is considered to be a risk factor of periodontitis. The biological association between both the diseases are under extensive studies for many years The knowledge of the two pathologies explores the two way relationship between diabetes and periodontitis.This paper aimed at reviewing the relationship between periodontitis and diabetes, and the impact of periodontal treatment on the glycemic control in diabetes. Furthermore, the control of periodontal disease may enhance glycemic control which contributes to a better control of periodontal disease.
\end{abstract}

Keywords: periodontal disease, inflammation, metabolic disorder, cytokines, glycemic control.

\section{Periodontitis}

Periodontitis is a slowly progressing inflammatory disease of tooth supporting structures which is irreversible in nature. It is initiated with gingivitis in which inflammation is confined to gingiva.The initial stage of gingivitis is a reversible condition, for the normal physiology of the tissue can be restored with good oral hygiene and removal of local factors [1]. Inflammation extending to connective tissues causes a slow destruction of supporting tissues of tooth. The first clinical sign of periodontal disease is the appearance of periodontal pockets which are caused by breakdown of collagen fibres of periodontal ligament.

These pockets offer a favourable niche for bacterial colonisation [2]. Advanced periodontitis is characterised by gingival erythema, bleeding, gingival recession, tooth mobility, gradual drifting of tooth, suppuration from pockets with tooth loss [1].

Periodontitis is a highly prevalent but largely hidden chronic disease. In the initial stages, this condition is typically asymptomatic and many patients are unaware until the condition has progressed enough to result in tooth mobility [3]. The periodontal disease causing bacteria that predominates the dental biofilm includes P.gingivalis, A. Actinomycetemcomitans, B.forsythus, and Fusobacterium nucleatum. According to the review of offenbacher in 1996 , the presence of bacteria in periodontal pocket triggers a reaction that starts with intervention of the neutrophil-compliment axis stimulating different cell types like neutrophils, macrophages, fibroblasts ,epithelial cells and others[4]. The cell walls of these gram negative bacteria made up of lipopolysaccharide and the endotoxins released acts as stimulants of host inflammatory responses[5] through Toll-like Protein receptors (TLRs).These receptors play an important role in the innate immune response , particularly in initial interaction between the infecting microorganism and phagocytic cells of monocyte lineage [6]. By means of chemotaxis, neutrophils reach the site of bacterial invasion and phagocytose the bacteria causing the host defence mechanism to release inflammatory mediators. The inflammatory mediators include prostaglandins, cytokines like IL-1alpha ,IL-beta, TNF-alpha and other mediators like RANKL and MMP. Cytokines like IL-1 and TNF alpha causes proliferation, differentiation and activation of osteoclast that causes resorption of bone. They are also involved in induction of protease tissue degrading. Production of prostaglandins by macrophages and fibroblasts causes induction of MMP and osteoclast.

MMP are matrix metallo protease released by macrophages, leukocytes and fibroblasts plays a pivotal role in degradation of extracellular matrix molecules such as collagen and elastin. They are responsible for breakdown of collagen and connective tissue attachments, which leads to deepening of pockets in periodontitis [7]. Other proteases includes elastase, cathepsin $G$ are capable of degrading the elastic and collagen fibres that supports the tooth. Further bone resorption in progressing periodontitis is caused by RANKL that are produced by fibroblasts, osteoblasts or lymphocytes. They cause differentiation and activation of osteoclasts and plays an important role in bone resorption. OPG (osteoprotegerin) is an inhibitor of RANKL which prevents binding of RANKL to specific receptors on osteoclasts precursor cells. Patients with advanced periodontitis are found to have higher levels of RANKL/ OPG ratio which serves as a biomarker that denotes the occurrence of periodontitis by indicating that molecular mechanisms of bone resorption are still active [8] .Such classical 
inflammatory pathway leads to the gradual mobility of the teeth which can be localised or generalised depending on the extension of the virulent bacteria and also other risk factors.

\section{Diabetes Mellitus}

Diabetes is a metabolic disorder characterised by impaired metabolism of glucose, lipids and proteins. The primary cause of type 1 diabetes is deficiency of insulin production which causes hyperglycaemia. It occurs normally due to autoimmune destruction of the beta- cells in the islets of langerhans of pancreas. Exposure to certain viral infections like mumps and coxackie viruses may also trigger abnormal antibody responses that damage beta cells [9] Type 2 is caused by impaired utilisation of insulin rather than insuffient insulin production. The major feature being lack of sensitivity to insulin by the body cells like fat and muscle cells which is mostly observed in obesity conditions. The classical complications seen in diabetic patients include atherosclerosis, nephropathy, neuropathy, and retinopathy and delayed wound healing. Whereas the minor symptoms seen in a diabetic patient orally are decreased salivary flow, xerostomia, chelosis and candidiasis [10] .The main cause of the severity of the disease is the weaker defensive mechanism in diabetes that leads the tissues to more prone to infections. Sustained hyperglycaemia causes increased levels of glucose in vascular system and even in the gingival tissue fluids. This excess glucose in body tends to attaches to the body molecules like proteins and lipids forming stable glucose protein product called amdori product which are reversible. But presence of a prolonged hyperglycaemic state in the body leads to its conversion into a highly stable accumulated glycation end products (AGE) that are irreversible in nature [10]. These AGE products interacts with monocytes and enhances chemo taxis which results in release of 4 times more inflammatory cytokines [11].

Delayed wound healing in diabetes is one condition that complicates the disease. The alteration of collagen metabolism by the glycosylation of the collagen molecules and vascular basement membrane thickening due to defective cross linking of collagen fibres causes narrowing of capillaries and henceforth delayed healing [12]. These cascades of events occur in periodontium of diabetic subjects leading to the alteration of host defensive cycle. The function of inflammatory cells gets altered in diabetic patients. The monocytes which play a significant part in periodontal tissue breakdown becomes hyper responsive to LPS (lipo polysaccharides). And also NADPH and glutamine production is found to decrease in diabetic patients, which are essential for rejuvenation of glutathione and activation of NADPH oxidase in neutrophils which are required for respiratory burst during migration, phagocytosis and apoptosis of neutrophils. Therefore the decreased utilization of glutamine and NADPH may contribute to impaired neutrophils function in diabetic patients [13].

This chronic metabolic disorder has a large impact on society by affecting over 100 millions people worldwide (Harris et all 1995)[14].

It is estimated that 194 million people suffered from diabetes across the globe in 2003, equalling $5.1 \%$ of world population. This is estimated to increase to 333 million or $6.3 \%$ of the world's population, in the year $2025[15]$.

\section{Pathogenic Mechanisms Linking Periodontitis And Diabetes}

Inflammation is a central feature of pathogenesis of diabetes and periodontitis [1] Periodontitis being an inflammatory disease is characterised by elevated levels of inflammatory mediators like TNF alpha, IL 6 and IL1beta, MMP- 8, MMP-9 and cytokines. These locally produced pro- inflammatory mediators move into the systemic circulation and alter the glucose and lipid metabolism and induce insulin resistance thus aggregating the condition [16].

Diabetes On the other hand is a manifestation of the hosts inflammatory response, because of cytokine induced acute phase response is closely involved in pathogenesis of this disease [17].

Diabetes and periodontitis are thus polygenic disorders with some degree of immune - regulatory dysfunction [18]. Furthermore the interaction between the two diseases has been proposed based of binding of AGE that are present in diabetic patients, to the receptors of macrophages [19]. In diabetic patients, AGE products are said to affect the migration and phagocytic activity of polymorphonuclear neutrophils, by binding to the macrophages, resulting in establishment of more pathogenic bacterial bio film, releasing a larger amount of cytokines and soluble mediators like TNF-alpha and IL-1 which mediates in tissue destruction of connective tissue and alveolar bone in periodontal disease though the cascade of release of hydrolase, MMPS and collagenase enzymes. Accumulation of AGE products in periodontium causes cross linking of collagen fibres which causes loss of integrity and breakdown of fibres [18].

Simultaneously, periodontal infection induces chronic state of insulin resistance, contributing the cycle of hyperglycaemia, and non enzymatic irreversible glycation. The cytokine production contributes to insulin resistance in a number of ways; by modifying the insulin receptor substrate-1 by serine phosphorylation and alteration of adipocyte function with increased production of free fatty acids there by participating in beta cell damage and burning out obesity in type 1 and 2 diabetes respectively [6] . 
Correlation between metabolic control and periodontal diseases also suggest that oxidative stress and inflammation exacerbates both pathologies [14].

\section{Association Between Periodontitis And Diabetes}

Evidence has consistently indicated that diabetes is a risk factor for increased severity of gingivitis and periodontitis. Conversely, periodontitis may be a risk factor for worsening glycemic control among patients with diabetes [20]. The first clear evidence to support this hypothesis came from investigations of individuals in the Gilia River Indian Community. Severe periodontitis was associated with an increased risk of poor glycemic control ( $\mathrm{HbA} 1 \mathrm{c}>9.0 \%)$ at follow up (minimum 2 years) suggesting that severe periodontitis was a risk factor for compromised diabetes management [21]. Although there are several risk factors for periodontitis, diabetes mellitus has been until now, the only systemic disease connected through biochemical mechanism with periodontal disease(Gheric et al 2008)[14] . Another fact related to the relationship between periodontitis and diabetes is majority of well controlled studies show a higher prevalence and severity of periodontal disease in diabetes than in non-diabetes with similar local irritation including greater loss of attachment, alveolar bone loss, bleeding gums on probing and tooth mobility [12].

Since 1990, a study of Puma Indians, a population that has the world's highest prevalence of type2 diabetes, showed a significant increase in prevalence of periodontal disease which indicates that diabetes is a risk factor for periodontal disease [22].

\section{Peridontal Treatment And Glycemic Control In Diabetes}

The extent of periodontal destruction in diabetic patients is influenced by glycemic control and the individual immune-regulatory capacity. Glycemic control can be improved with help of scaling and root planning combined with short term anti microbial therapy, especially in patients with advanced periodontal disease and poor metabolic control [14]. A number of recent studies evaluated whether the use of systemic antibiotics improved periodontal prognosis and metabolic control in diabetic patients. Mechanical periodontal therapy with $100 \mathrm{mg}$ doxycycline produced a $0.6 \%$ reduction in HbAlc in type 2 diabetic patients; doxycycline appears to be a potent modifier of the response to periodontal treatment in diabetic patients by inhibiting non enzymatic glycosylation and metalloproteinase activity [2].

Results from the landmark diabetes control and complication Trial (type 1 diabetes) and the UK prospect Diabetes study (UKPDS)(type 2 diabetes) demonstrated that attaining and maintaining good glycemic control could reduce the risk for and slow the progression of microvascular complication in patients with type 1 and type 2 diabetes [18].

\section{Conclusion}

Periodontitis is the most common and prevalent disease affecting $40-60 \%$ of adults but largely hidden. Because periodontal disease are silent in nature, most patients do not realise they have such condition until significant destruction has occurred [18]. Treating periodontal infection in people with diabetes is an important component in maintaining oral health. It may also have an important role in establishing and maintaining glycemic control and also in delaying onset or progression of diabetic complication.

[1] P.M. Preshaw , A.L.Alba , D.Herrer, S.Jepsen et al ,periodontitis and diabetes: a two-way relationship; Diabetologia(2012)55:2131

[2] Antonio Bascones-Martinez et al, periodontal diseases and diabetes- Review of literature, Med Oral Patol Oral Cir Bucal. 2011 sep 1;16(6):e722-9.

[3] O'Dowd LK et al, patients' experiences of the impact of periodontal disease .journal of clinical periodontology. (2010), 37:334339

[4] Negrato CA, Tarzia o Jovanovic 1 ,Chinellato lem ,Periodontal disease and diabetes mellitus , Journal of Appl Oral Sci.(2013);21(1):1-12

[5] Francis G.Serio, Teresa B.Duncan, The pathogenesis and treatment of periodontal diseases, The Acadamy of dental Therapeutics and Stomatology.

[6] Janet H. Southerland, George W. Taylor, Steven Offenbacher, Diabetes and Periodontal infection: Making the connection , Clinical Diabetes(2005), 23(4);171-178

[7] Nitin Saroch, periobasics, Basic periodontology, chapter 12. Matrix Mettaloproteinase (MMP's) and their role in pathogenesis of periodontal diseases.

[8] Belibasakis GN,et al.The RANKL-OPG system in clinical periodontology. journal of clinical periodotology.2012;39(3):239-48.

[9] Debora C. Matthews,The two-way relationship Between Diabetes and Periodontal Disease, Journal of the Canadian Dental Association, 2002;68(3):161-164.

[10] J Deshmukh, M Basnaker, VK Kulkarni, Katti G, Periodontal disease and Diabetes- A Two Way Street Dual Highway?, Peoples Journal Of Scientific Research, July 2011;vol. 4(2).

[11] E Hatanaka ,P T Monteagudo, A Campa.Neutrophils and monocytes as potentially important sources of proinflammatory cytokines in diabetes.Clinical and Expeimental Immunology.2006;146(3):443-447.

[12] Rajkumar Daniel, subramanium Gokulanathan,Thangavelu Kavin.Diabetes and periodontal diseases.Jof Pharmacy and Bioallied Sciences 2012.4(2)S280-S282. 
[13] Harpreet Singh Grover, Shailly Luthra, Molecular mechanisms involved in the bidirectional relationship between diabetes mellitus and periodontal disease, journal of Indian Society of Periodontology(2013), 17(3),292-301.

[14] Oana A.Velea et al, Diabetes And Periodontal Disease- A Two Way Road:current concepts and future considerations, European Scientific Journal March 2013. Vol.9,No.9.

[15] Nesse et al, Dose-response relationship between periodontal inflamed surface area and HbA1c in type 2 Diabetes,Journal of Clinical Periodontology (2009)10.1111/j.1600-051X.2009.01377.x.

[16] Nogueira-Filho, Impact of Periodontitis on the Diabetes-Related inflammatory status,Journal of the Cannadian Dental Association (2010);76:a35.

[17] Pickup JC. Inflammation and activated innate immunity in the pathogenesis of type 2 diabetes. Diabetes Care. 2004;27(3):813-23.

[18] Gavrav Malik , Gurvanit Lehl, Manjit Talwar. Association of periodontitis with Diabetes Mellitus:a review. Journal of Medical college Chandigarh, 2011,vol.1,No.1.

[19] Grossi SG,Genco RJ.Periodontal disease and diabetes mellitus :a two way relationship. Ann Periodontol. 1998;3:51-61.

[20] Salvi GE, Carollo-Bittel B,Lang NP.Effects of diabetes mellitus on periodontal and peri-implant condition.Update on association and risks.Journal of Clinical Periodontology.2008;35(S-8):398-409.

[21] Taylor GW, Burt BA,Becker MP et al.Severe periodontitis and risk for poor glycemic control in patients with non-insulindependent diabetes mellitus. J Periodontol (1996).67:1085-1093.

[22] Cordon Nikiforuk.Diabetes and periodontal disease - a complex two-way connection.Diabetes Care News, 2004.19;1-4. 